\title{
A REPRESENTATION THEOREM OF THE SPHERICAL WAVE FUNCTIONS
}

\author{
Meryem KAYA
}

Gazi University, Faculty of Arts and Sciences, Department of Mathematics Ankara, TURKEY

Abstract-Let $\phi_{i}^{*}$ and $\psi_{i}(i=0,1, \ldots, n-1)$ are the solutions of the equations $\left(\square^{2}-\frac{\mathrm{n}-1}{\mathrm{r}^{2}}\right) \phi_{\mathrm{i}}^{*}=0$ and $\square^{2} \psi_{\mathrm{i}}=0$ respectively. In this paper it is shown that if $\mathrm{u}$ and $\mathrm{v}$ are satisfied the equations $\left(\square^{2}-\frac{n-1}{r^{2}}\right)^{n} u=0$ and $\square^{2 n} v=0$ respectively then $u$ and $v$ have the representations $\mathrm{u}=\phi_{0}^{*}+\mathrm{t} \phi_{1}^{*}+\ldots+\mathrm{t}^{\mathrm{n}-1} \phi_{\mathrm{n}-1}^{*}$ and $\mathrm{v}=\psi_{0}+\mathrm{t} \psi_{1}+\ldots+\mathrm{t}^{\mathrm{n}-1} \psi_{\mathrm{n}-1}$ where $\square^{2}=\frac{1}{\mathrm{r}^{\mathrm{n}-1}} \frac{\partial}{\partial \mathrm{r}}\left(\mathrm{r}^{\mathrm{n}-1} \frac{\partial}{\partial \mathrm{r}}\right)-\frac{\partial^{2}}{\partial \mathrm{t}^{2}}$.

\section{INTRODUCTION}

The wave equation

$\frac{\partial^{2} u}{\partial x_{1}^{2}}+\cdots+\frac{\partial^{2} u}{\partial x_{n}^{2}}-\frac{\partial^{2} u}{\partial t^{2}}=0$

is the most important for partial differential equation of the hyperbolic type. In equation (1), $\mathrm{x}=\left(\mathrm{x}_{1}, \ldots, \mathrm{x}_{\mathrm{n}}\right)$ are called the space variables in $\mathrm{R}^{\mathrm{n}}$ and $\mathrm{t}$ is the time variable. Using the spherical coordinates in the $n$-dimensional space $\mathrm{R}^{\mathrm{n}}$, the wave equation (1) takes the form

$\frac{1}{r^{n-1}} \frac{\partial}{\partial r}\left(r^{n-1} \frac{\partial u}{\partial r}\right)-\frac{\partial^{2} u}{\partial t^{2}}=0$

which depends only on $r$ and $t$ and does not depend on angular variables. Equation (2) is known as the equation of spherical waves [7].

Almansi proved a theorem concerning the representation of the general solution of the equation $\nabla^{2 n} F=0$ [1]. Eubanks and Sternberg generalized the Almansi's theorem further but their theorems hold true only on condition that a region is convex in the $\mathrm{z}$ direction [2]. M.Z. Wang and X.S. Xu give two kinds of representation of the general solution of the equation $\nabla^{2 \mathrm{n}} \mathrm{F}=0$ for the case in which $\nabla^{2}$ is an axisymmetric threedimensional Laplacian operator without the supposition of convexity in [6]. Now firstly some Lemmas and then some expansions for the solutions of the above equations are given. 
Let the region is a surfaces of revolution whose axis coincides with the $t$-axis $t>0$ and we denote the intersection of its interior with the open section plane

$r=\sqrt{\sum_{i=1}^{n} x_{i}^{2}}>0, \theta_{1}=0$ by D where

$\mathrm{x}_{1}=\mathrm{r} \cos \theta_{1}$,

$x_{2}=r \sin \theta_{1} \cos \theta_{2}$,

$x_{n-2}=r \sin \theta_{1} \ldots \sin \theta_{n-3} \cos \theta_{n-2}$,

$x_{n-1}=r \sin \theta_{1} \ldots \sin \theta_{n-2} \cos \theta_{n-1}$,

$x_{n}=r \sin \theta_{1} \ldots \sin \theta_{n-2} \sin \theta_{n-1}$,

$0<\theta_{1}<\pi, \ldots, 0<\theta_{n-2}<\pi, 0<\theta_{n-1}<2 \pi$

Lemma 1. If the functions $\phi^{*}$ and $\phi$ satisfy the equation

$\frac{1}{r^{n-1}} \frac{\partial}{\partial r}\left(r^{n-1} \phi^{*}\right)=\frac{\partial \phi}{\partial t}$

$\frac{\partial \phi^{*}}{\partial \mathrm{t}}=\frac{\partial \phi}{\partial \mathrm{r}}$

then

$\left(\square^{2}-\frac{\mathrm{n}-1}{\mathrm{r}^{2}}\right) \phi^{*}=0$

$\square^{2} \phi=0$

Proof. Direct computation yields (4) and (5) from (3).

Lemma 2. Suppose that $\mathrm{D}$ is simply connected. Then

$i$. If a function $\phi^{*}(\mathrm{r}, \mathrm{t})$ in D satisfies (4), there exists $\phi(\mathrm{r}, \mathrm{t})$ in D such that equations (3) hold true.

ii. If a function $\phi(r, t)$ in D satisfy (5) there exists $\phi^{*}(r, t)$ in D such that equations hold true.

Proof. i. Let

$\phi(r, t)=\int_{\left(r_{0}, t_{0}\right)}^{(r, t)} \frac{\partial \phi^{*}}{\partial t} d r+\frac{1}{r^{n-1}} \frac{\partial}{\partial r}\left(r^{n-1} \phi^{*}\right) d t$

where $\left(\mathrm{r}_{0}, \mathrm{t}_{0}\right)$ is an arbitrary fixed point of $\mathrm{D}$. In view of equation (4) and the simple connectivity of $\mathrm{D}$, the line integral of equation (6) is independent of the path. Thus, the 
function $\phi(r, t)$ is single valued in D. It is obvious from equation (6) that equation (3) hold.

ii. Similarly, we take

$r^{n-1} \phi^{*}(r, t)=\int_{\left(r_{0}, t_{0}\right)}^{(r, t)} r^{n-1} \frac{\partial \phi}{\partial t} d r+r^{n-1} \frac{\partial \phi}{\partial r} d t$

The line integral of equation (7) is also independent of the path from the reason which is mentioned above. On the other hand it is clear from equation (7) that equation (3) hold.

Lemma 3. Suppose that D is simply connected. If $\phi^{*}$ and $\phi$ satisfy equation (3) then there exist another pair of functions $\mathrm{A}^{*}$ and $\mathrm{A}$ satisfy equation (3) and such that

$$
\begin{aligned}
& \frac{1}{r^{n-1}} \frac{\partial}{\partial r}\left(r^{n-1} A^{*}\right)=\frac{\partial A}{\partial t}=\phi \\
& \frac{\partial A^{*}}{\partial t}=\frac{\partial A}{\partial r}=\phi^{*}
\end{aligned}
$$

Proof. Let

$r^{n-1} A^{*}=\int_{\left(r_{0}, t_{0}\right)}^{(r, t)} r^{n-1} \phi d r+r^{n-1} \phi^{*} d t$

$\mathrm{A}=\int_{\left(\mathrm{r}_{0}, \mathrm{t}_{0}\right)}^{(\mathrm{r}, \mathrm{t})} \phi^{*} \mathrm{dr}+\phi \mathrm{dt}$

where $\left(r_{0}, t_{0}\right)$ is an arbitrary fixed point of D. From (3) the line integrals of $(9)$ are independent of path. It is obvious from (9) that equations (8) hold.

\section{SOME EXPANSIONS FOR SPHERICAL WAVE FUNCTIONS}

In this section we prove the following theorems.

Theorem 1. Let $u(r, t)$ and $v(r, t)$ satisfy

$$
\begin{array}{ll}
\left(\square^{2}-\frac{\mathrm{n}-1}{\mathrm{r}^{2}}\right)\left(\square^{2}-\frac{\mathrm{n}-1}{\mathrm{r}^{2}}\right) \mathrm{u}=0 & \text { in } \mathrm{D} \\
\square^{2} \square^{2} \mathrm{v}=0 & \text { in } \mathrm{D}
\end{array}
$$

Then

$$
\begin{aligned}
& u=\phi_{01}^{*}+\frac{1}{2} t \phi_{1}^{*} \\
& u=\phi_{02}^{*}+\frac{1}{2} r \phi_{2}
\end{aligned}
$$

and

$\mathrm{v}=\psi_{01}+\frac{1}{2} \mathrm{r} \phi_{2}^{*}$ 
$\mathrm{v}=\psi_{02}+\frac{1}{2} \mathrm{t} \phi_{2}$

where $\phi_{i}^{*}$ and $\phi_{\mathrm{i}}(\mathrm{i}=1,2)$ satisfy equation (3) and $\phi_{01}^{*}, \phi_{02}^{*}, \psi_{01}$ and $\psi_{02}$ satisfy

$\begin{array}{ll}\left(\square^{2}-\frac{\mathrm{n}-1}{\mathrm{r}^{2}}\right) \phi_{0 \mathrm{i}}^{*}=0 & \mathrm{i}=1,2 \\ \square^{2} \psi_{0 \mathrm{i}}=0 & \mathrm{i}=1,2\end{array}$

Proof. Let

$\mathrm{p}_{i}^{*}=\left(\square^{2}-\frac{\mathrm{n}-1}{\mathrm{r}^{2}}\right) \mathrm{u}$

$\mathrm{p}_{2}=\square^{2} \mathrm{v}$.

So that

$\left(\square^{2}-\frac{\mathrm{n}-1}{\mathrm{r}^{2}}\right) \mathrm{p}_{1}^{*}=0$

$\square^{2} \mathrm{p}_{2}=0$

since $\mathrm{p}_{1}^{*}$ and $\mathrm{p}_{2}$ satisfy $(20)$ and (21), according to Lemma 2 , there exist $\mathrm{p}_{1}$ and $\mathrm{p}_{2}^{*}$ which satisfy

$\frac{1}{r^{n-1}} \frac{\partial}{\partial r}\left(r^{n-1} p_{i}^{*}\right)=\frac{\partial p_{i}}{\partial t} \quad(i=1,2)$

$\frac{\partial \mathrm{p}_{\mathrm{i}}^{*}}{\partial \mathrm{t}}=\frac{\partial \mathrm{p}_{\mathrm{i}}}{\partial \mathrm{r}} \quad(\mathrm{i}=1,2)$.

From (22), $\mathrm{p}_{\mathrm{i}}^{*}$ and $\mathrm{p}_{\mathrm{i}}(\mathrm{i}=1,2)$ satisfy equation (3). According to Lemma 3, there exist $\phi_{\mathrm{i}}$ and $\phi_{i}^{*}(\mathrm{i}=1,2)$ that satisfy

$$
\begin{array}{ll}
\frac{1}{\mathrm{r}^{\mathrm{n}-1}} \frac{\partial}{\partial \mathrm{r}}\left(\mathrm{r}^{\mathrm{n}-1} \phi_{i}^{*}\right)=\frac{\partial \phi_{\mathrm{i}}}{\partial \mathrm{t}}=\mathrm{p}_{\mathrm{i}} & (\mathrm{i}=1,2) \\
\frac{\partial \phi_{i}^{*}}{\partial \mathrm{t}}=\frac{\partial \phi_{\mathrm{i}}}{\partial \mathrm{r}}=\mathrm{p}_{\mathrm{i}}^{*} & (\mathrm{i}=1,2)
\end{array}
$$

Then

$$
\begin{aligned}
& \left(\square^{2}-\frac{n-1}{r^{2}}\right) u=\frac{\partial \phi_{1}^{*}}{\partial t}=\frac{\partial \phi_{1}}{\partial r} \\
& \square^{2} v=\frac{1}{r^{n-1}} \frac{\partial}{\partial r}\left(r^{n-1} \phi_{2}^{*}\right)=\frac{\partial \phi_{2}}{\partial t}
\end{aligned}
$$


$\left(\square^{2}-\frac{\mathrm{n}-1}{\mathrm{r}^{2}}\right)\left(\mathrm{u}+\frac{1}{2} \mathrm{t} \phi_{1}^{*}\right)=0$

$\left(\square^{2}-\frac{n-1}{r^{2}}\right)\left(u-\frac{1}{2} r \phi_{1}\right)=0$

$\square^{2}\left(\mathrm{v}+\frac{1}{2} \mathrm{t} \phi_{2}\right)=0$

$\square^{2}\left(\mathrm{v}-\frac{1}{2} \mathrm{r} \phi_{2}^{*}\right)=0$

we have equations (12)-(15) from equations (26)-(29). The proof is completed.

To generalize further, we have the following theorem.

Theorem 2. Let the functions $u(r, t)$ and $v(r, t)$ satisfy

$\begin{array}{ll}\left(\square^{2}-\frac{\mathrm{n}-1}{\mathrm{r}^{2}}\right)^{\mathrm{n}} \mathrm{u}=0 & \text { in } \mathrm{D} \\ \square^{2 \mathrm{n}} \mathrm{v}=0 & \text { in } \mathrm{D}\end{array}$

then

$\mathrm{u}=\phi_{0}^{*}+\mathrm{t} \phi_{1}^{*}+\ldots+\mathrm{t}^{\mathrm{n}-1} \phi_{\mathrm{n}-1}^{*}$

$\mathrm{v}=\psi_{0}+\mathrm{t} \psi_{1}+\ldots+\mathrm{t}^{\mathrm{n}-1} \psi_{\mathrm{n}-1}$

$\left(\square^{2}-\frac{\mathrm{n}-1}{\mathrm{r}^{2}}\right) \phi_{\mathrm{i}}^{*}=0 \quad \mathrm{i}=0, \ldots, \mathrm{n}-1$

$\square^{2} \psi_{\mathrm{i}}=0 \quad \mathrm{i}=0, \ldots, \mathrm{n}-1$

Proof. We use induction. It is clear that equations (32) and (33) hold for $n=1$ and $n=2$. Next by induction, we assume equations (32) and (33) hold for $n \leq k$ and show them hold for $n=k+1$. Now assume that

$\left(\square^{2}-\frac{\mathrm{n}-1}{\mathrm{r}^{2}}\right)^{\mathrm{k}+1} \mathrm{u}=\left(\square^{2}-\frac{\mathrm{n}-1}{\mathrm{r}^{2}}\right)^{k}\left[\left(\square^{2}-\frac{\mathrm{n}-1}{\mathrm{r}^{2}}\right) \mathrm{u}\right]=0$

$\square^{2(\mathrm{k}+1)} \mathrm{v}=\square^{2 \mathrm{k}} \square^{2} \mathrm{v}=0$

By induction, we have

$\left(\square^{2}-\frac{\mathrm{n}-1}{\mathrm{r}^{2}}\right) \mathrm{u}=\mathrm{A}_{0}^{*}+\mathrm{tA}_{1}^{*}+\ldots+\mathrm{t}^{\mathrm{k}-1} \mathrm{~A}_{\mathrm{k}-1}^{*}$

$\square^{2} v=B_{0}+t B_{1}+\ldots+t^{k-1} B_{k-1}$

where 
$\left(\square^{2}-\frac{\mathrm{n}-1}{\mathrm{r}^{2}}\right) \mathrm{A}_{i}^{*}=0$

$\mathrm{i}=0,1, \ldots \mathrm{k}-1$

$\square^{2} \mathrm{~B}_{\mathrm{i}}=0$ $\mathrm{i}=0,1, \ldots \mathrm{k}-1$

According to Lemma 2 and 3, we have

$2 \mathrm{k} \frac{\partial \phi_{\mathrm{k}}^{*}}{\partial \mathrm{t}}=-\mathrm{A}_{\mathrm{k}-1}^{*}$

$2(\mathrm{~m}-1) \frac{\partial \phi_{\mathrm{m}-1}^{*}}{\partial \mathrm{t}}=-\mathrm{A}_{\mathrm{m}-2}^{*}-\mathrm{m}(\mathrm{m}-1) \phi_{\mathrm{m}}^{*} \mathrm{~m}=\mathrm{k}, \mathrm{k}-1, \ldots, 2$

$2 \mathrm{k} \frac{\partial \psi_{\mathrm{k}}}{\partial \mathrm{t}}=-\mathrm{B}_{\mathrm{k}-1}$

$2(\mathrm{~m}-1) \frac{\partial \psi_{\mathrm{m}-1}}{\partial \mathrm{t}}=-\mathrm{B}_{\mathrm{m}-2}-\mathrm{m}(\mathrm{m}-1) \psi_{\mathrm{m}} \quad \mathrm{m}=\mathrm{k}, \mathrm{k}-1, \ldots, 2$

where $\phi_{i}^{*}$ and $\psi_{i}(i=1,2, \ldots, k)$ satisfy $(34)$.

From equations (37)-(40) it is not difficult to obtain the following

$\left(\square^{2}-\frac{\mathrm{n}-1}{\mathrm{r}^{2}}\right)\left(\mathrm{u}-\mathrm{t} \phi_{1}^{*}-\mathrm{t}^{2} \phi_{2}^{*}-\ldots-\mathrm{t}^{\mathrm{k}} \phi_{\mathrm{k}}^{*}\right)=0$

$\square^{2}\left(\mathrm{v}-\mathrm{t} \psi_{1}-\mathrm{t}^{2} \psi_{2}-\ldots-\mathrm{t}^{\mathrm{k}} \psi_{\mathrm{k}}\right)=0$

Therefore

$\mathrm{u}=\phi_{0}^{*}+\mathrm{t} \phi_{1}^{*}+\mathrm{t}^{2} \phi_{2}^{*}+\ldots+\mathrm{t}^{\mathrm{k}} \phi_{\mathrm{k}}^{*}$

$\mathrm{v}=\psi_{0}+\mathrm{t} \psi_{1}+\mathrm{t}^{2} \psi_{2}+\ldots+\mathrm{t}^{\mathrm{k}} \psi_{\mathrm{k}}$

where

$\left(\square^{2}-\frac{\mathrm{n}-1}{\mathrm{r}^{2}}\right) \phi_{0}^{*}=0$

$\square^{2} \psi_{0}=0$

By induction the proof is completed. 


\section{REFERENCES}

1. Almansi, E. Sull'integrazione dell' equazione differenziale $\nabla^{2 n} \mathrm{~F}=0$. Ann. di. Mat.Ser 3, $2,1,1899$

2. Eubanks, R.A. and Sternberg, E. On the axisymmetric problem of elasticity theory for a medium with transverse isotropy. J.Rat.Mech.Anal. 3, 89-101, 1954.

3. Carlson, D.E. A note on the solutions of Boussinesq, Love and Marguerre in axisymmetric elasticity. J. Elasticity 13, 345-349, 1983.

4. Courant, R. and Hilbert, D. Methods of Mathematical Physics, V.II, John Wiley, 1962.

5. Garabedian,P.R. Partial Differential Equations, John Wiley, 1964.

6. Wang, M.Z and Xu,X.S. A generalization of Almansi's theorem and its application. Appl Math. Modelling 14, 275-279, 1990.

7. Zachmanoglou, E.C. and Thoe, Dale W. Introduction to Partial Differential Equnations with Applications, Dover Publications, 1986. 